\title{
Entre barreras y facilitadores: las experiencias de los estudiantes universitarios con discapacidad
}

\section{Between barriers and enablers: The experiences of university students with disabilities}

\author{
Judith PÉREZ-CASTRO*
}

El objetivo de este artículo es analizar algunas barreras que enfrentan los estudiantes con discapacidad en la educación superior. El trabajo se deriva de una investigación desarrollada en dos instituciones: la Universidad Nacional Autónoma de México y la Universidad Juárez Autónoma de Tabasco. El método fue el estudio de caso, con entrevistas en profundidad a estudiantes con discapacidad y revisión de documentos institucionales e información estadística sobre esta población. Los resultados mostraron que los alumnos enfrentan barreras estructurales, como la realización de los trámites institucionales y la dificultad para que los profesores ajusten los contenidos y las actividades de enseñanza; ambientales, como la poca accesibilidad a las instalaciones institucionales y al material bibliográfico; y actitudinales, como la escasa sensibilización de los profesores, del personal administrativo y de sus compañeros. La conclusión apunta a la necesidad de cambiar las culturas, las políticas y las prácticas institucionales a través de estrategias integrales que permitan ampliar las oportunidades educativas de estos estudiantes.

The objective of this paper is to analyze the barriers faced by students with disabilities in higher education. This paper is part of a research carried out in two institutions: The National Autonomous University of Mexico and the Juarez Autonomous University of Tabasco. It was a case study research and, as part of it, we interviewed a group of students with disabilities and, at the same time, analyzed institutional policies on inclusion and statistical data. Results showed that students face structural barriers, for example, difficulties to complete institutional procedures and to adjust syllabus contents and learning activities; environmental barriers, such as the lack of accessibility to institutional facilities and to specialized literature; and attitudinal barriers, for instance, the unawareness of teachers, administrative staff and classmates. We conclude that it is necessary to change cultures, policies and institutional practices, through comprehensive strategies in order to increase educational opportunities for these students.

\section{Palabras clave:}

barreras, facilitadores, educación superior, inclusión, discapacidad

\section{Keywords:}

barriers, enablers, higher education, inclusion, disability

Recibido: 13 de febrero de 2019. | Aceptado para su publicación: 25 de junio de 2019. Recuperado de: https://sinectica.iteso.mx/index.php/SINECTICA/article/view/948 doi: 10.31391/S2007-7033(2019)0053-003

Sección: Investigaciones temáticas

* Doctora en Ciencia Social con especialidad en Sociología. Investigadora en el Instituto de Investigaciones sobre la Universidad y la Educación-UNAM. Líneas de investigación: inclusión y equidad en educación, políticas educativas, ética y valores profesionales. Correo electrónico: pkjudith33@yahoo.com.mx/jcperez@unam.mx/ http://orcid. org/0000-0002-5176-0531 


\section{INTRODUCCIÓN}

$\mathrm{D}$ e acuerdo con la Organización Mundial de la Salud (OMS, 2001), las barreras para la inclusión son todos aquellos factores físicos, sociales y actitudinales que impiden o limitan la realización plena de los individuos. Se trata de un concepto dinámico porque remite a la interacción entre las personas y las condiciones de su entorno, es decir, los objetos, normas, procedimientos o actitudes no son por sí mismos una barrera y, por lo tanto, los podemos modificar (Thomas, 2014).

El concepto de barreras para la inclusión fue el resultado de la lucha de diferentes colectivos de personas con discapacidad, en especial de la Unión de Personas Físicamente Impedidas contra la Segregación (UPIAS por sus siglas en inglés), que sentaron las bases políticas e intelectuales de lo que luego se conocería como el modelo social de discapacidad (Shakespeare, 2013). Los planteamientos de la UPIAS cuestionaban la perspectiva médico-rehabilitadora que, hasta los años setenta, había predominado en el estudio de la discapacidad y la provisión de los servicios, con el argumento de que era la sociedad la que generaba contextos discapacitantes (Shakespeare \& Watson, 2001). La idea era que, al centrar la atención en el medio y no en las personas, se incrementaban las posibilidades de develar las diferentes barreras y formas de exclusión, así como de diseñar estrategias para su desmantelamiento.

De acuerdo con Oliver (1996), detrás de todo esto había una impronta de cambio social y político, sobre todo en la vertiente británica del modelo social. En los principios fundamentales de la discapacidad, la UPIAS sostenía que la discapacidad era un proceso impuesto socialmente ante los impedimentos físicos y sociales, que excluían a las personas de su participación plena (Shakespeare, 2013); es decir, se consideraban un grupo socialmente oprimido, por lo que la meta era desafiar la posición que, por tradición, se les había atribuido, como enfermos, inútiles, desafortunados, diferentes y sometidos (Hunt, 2017).

En la vertiente norteamericana, las personas con discapacidad se veían a sí mismas como un grupo minoritario; por ello, su interés se centró más en la promulgación de leyes contra la discriminación que en la relación entre las dimensiones biológica y social de la discapacidad (Shakespeare \& Watson, 2001). Con la distinción entre discapacidad e impedimento, el modelo social contribuyó de manera fundamental al desarrollo de las políticas para la eliminación de las barreras para la inclusión y la participación (Swain \& French, 2014).

En este artículo, analizamos las barreras y los facilitadores que intervienen en los aprendizajes y las experiencias de un grupo de estudiantes universitarios con discapacidad. Iniciamos con la discusión de las barreras para la inclusión social para, después, centrarnos en aquellas relacionadas con la educación y, de manera concreta, con la educación superior. Nos interesa destacar también la relación entre barreras y facilitadores, así como algunos de los rasgos que los caracterizan, específicamente la reversibilidad, el efecto de refuerzo y la extensibilidad. Los resultados se organizan en tres grandes apartados: barreras estructurales, ambientales y actitudinales. Al final, presentamos las consideraciones generales sobre los aspectos aquí abordados.

Pérez. Entre barreras y facilitadores: las experiencias de los estudiantes universitarios con discapacidad Sinéctica 53 www.sinectica.iteso. $m x$ 


\section{BARRERAS SOCIALES PARA LA PARTICIPACIÓN DE LAS PERSONAS CON DISCAPACIDAD}

Una de las metas de la inclusión, además de reconocer el valor de la diversidad humana y fortalecer la participación de las personas en los distintos ámbitos de la vida social, es identificar y reducir las barreras que obstaculizan su desarrollo personal y colectivo (Booth \& Ainscow, 2002). Como hemos dicho, la noción de barreras apunta a las condiciones del medio que restringen el desarrollo pleno; de la interacción entre las capacidades de las personas y el contexto, incluyendo las barreras y los facilitadores, resultan las distintas experiencias de la discapacidad (ONU, 2006).

De las diferentes clasificaciones sobre las barreras, en este trabajo retomamos, principalmente, la tipología de French (2017), que distingue entre:

1. Barreras estructurales -que se refieren a las normas subyacentes, costumbres e ideología de las organizaciones e instituciones, las cuales se basan en juicios de 'normalidad' y se sustentan en jerarquías de poder.

2. Barreras ambientales -que se refieren a las barreras físicas dentro del medio ambiente, por ejemplo, escalones, agujeros en el pavimento y la falta de recursos para las personas con discapacidad, por ejemplo, la falta de señalización en Braille y la carencia de intérpretes de lengua de señas. También, se refiere a las formas en que se hacen las cosas, que pueden excluir a las personas con discapacidad, por ejemplo, la forma en que se llevan a cabo las reuniones y el tiempo destinado para hacer las tareas.

3. Barreras actitudinales -que se refieren a las actitudes y comportamientos adversos hacia las personas con discapacidad.

[1. Structural barriers -which refer to the underlying norms, mores and ideologies of organisations and institutions which are based on judgments of 'normality' and which are sustained by hierarchies of power.

2. Environmental barriers -which refer to physical barriers within the environment, for example steps, holes in the pavement, and lack of resources for disabled people, for example lack of braille and lack of sign language interpreters. It is also referred to the ways things are done which may exclude disabled people, form example the way meetings are conducted and the time allowed for tasks.

3. Attitudinal barriers -which refer to the adverse attitudes and behavior of people towards disabled people.] (Traducción propia, p.11.)

Las barreras, al cruzarse con las desigualdades estructurales (Subirats, Gomà y Brugué, 2005), como la pobreza, la falta de servicios públicos, la fragmentación social y la carencia de marcos jurídicos incluyentes, profundizan las desventajas de estas personas y elevan su grado de vulnerabilidad ante otros riesgos. Parafraseando a Young (2001), podríamos decir que las barreras son el resultado de ideas y acciones colectivas condicionadas sociohistóricamente, las cuales tienen efecto en el medio físico y cultural, desde la organización de las ciudades hasta los espacios que hemos dejado para otros seres de la naturaleza.

De este modo, las barreras -y su contraparte: los facilitadores- se encuentran distribuidas casi en todos los ámbitos de la vida social. En la salud, por ejemplo, se ha visto que la organización y las normas que sustentan los sistemas pueden resultar muy difíciles para las personas con discapacidad y sus familias (OMS, 2011), como

Pérez. Entre barreras y facilitadores: las experiencias de los estudiantes universitarios con discapacidad Sinéctica 53 www.sinectica.iteso.mx 
los horarios para la asignación de turnos, las enormes listas o filas de espera, el alza en los costos, la complejidad de los trámites para ser referidos a servicios especializados, las actitudes de los médicos, enfermeras y trabajadores de apoyo, y las largas distancias entre los centros de atención y los lugares de residencia de los sujetos. $\mathrm{Si}$ bien todos los que recibimos atención pública tenemos que lidiar con estas barreras organizativas, en la población con discapacidad sus efectos se acrecientan y disminuyen sus posibilidades de acceso a los servicios o de un tratamiento adecuado.

El mercado laboral es otro ámbito en el que se han observado distintas barreras. Bennett, Ray y Wilson (2016) han identificado que las más frecuentes son las actitudes de los empleadores hacia la población con discapacidad, requisitos laborales y procesos de reclutamiento complicados, y la inaccesibilidad física. A esto se suma la escasez de vacantes adecuadas, los patrones y ritmos de trabajo (Grover \& Piggott, 2015), la falta de habilidades laborales y la poca confianza que las propias personas con discapacidad tienen sobre sí mismas (Bennett, Ray \& Wilson, 2016).

En cuanto al acceso a la información y la comunicación, la OMS (2011) señala que, aunque ha habido avances en cuanto a la tecnología asistida, la información impresa sigue siendo el principal recurso en muchos países. Las barreras más frecuentes tienen que ver con el formato y los contenidos de la información, el desarrollo de software y dispositivos tecnológicos adaptados, la prestación de los servicios de televisión e internet, y la asistencia técnica (Simpson, 2009).

Finalmente, un aspecto poco explorado es la cultura y la recreación. Hay una tendencia más o menos generalizada a asociar la discapacidad con la rehabilitación terapéutica, pero es menos frecuente pensar en otro tipo de actividades. La disponibilidad de los padres, tutores o cuidadores, la carencia de políticas y programas recreativos, deportivos y culturales incluyentes, la poca accesibilidad de las instalaciones, y la falta de personal capacitado son algunas de las barreras más recurrentes (Shields \& Synnot, 2016). Otras se derivan del desinterés, el miedo y los tiempos de las personas con discapacidad, la falta de apoyo o la sobreprotección de los familiares, la limitada información sobre las actividades y programas, los costos, el transporte y las actitudes negativas hacia la discapacidad (Serrano et al. 2013).

\section{LA INCLUSIÓN Y LAS BARRERAS EN LA EDUCACIÓN SUPERIOR}

El modelo social cambió la forma de ver la discapacidad, al trasladar la discusión de las limitaciones de las personas a la forma en que esta se producía socialmente (Hughes y Paterson, 2008). De la misma manera, en la educación, la noción de barreras desplazó la atención de las "necesidades educativas especiales" hacia los factores que obstaculizaban los aprendizajes y la participación de las personas con discapacidad (Booth \& Ainscow, 2002).

El giro hacia las barreras va más allá del uso de términos "políticamente correctos", lo que se busca es ampliar el debate hacia los temas del derecho a la educación, los factores de exclusión y el fracaso escolar. En su defensa del enfoque de inclusión, Echeita y Ainscow (2011) sostienen que este es un proceso que requiere la identificación y eliminación de las barreras, porque son ellas "las que impiden el ejercicio efectivo de los derechos, en este caso, a una educación inclusiva” (p. 33).

Pérez. Entre barreras y facilitadores: las experiencias de los estudiantes universitarios con discapacidad Sinéctica 53 www.sinectica.iteso.mx 
Para estos autores, las barreras se encuentran en toda la organización escolar, en las culturas, las políticas y las prácticas educativas. Entre las más importantes, están las creencias de los profesores, la formación inadecuada, el predominio de concepciones de la educación poco inclusivas y de currículos restrictivos, la prevalencia de métodos de enseñanza "transmisivos" y la falta de recursos para la inclusión (Echeita y Ainscow, 2011).

Otra forma de acercarse a las barreras es desde el modelo biopsicosocial, desarrollado por la OMS (2001), que distingue entre las de tipo sistémico y las relacionadas con la dinámica escolar. Las primeras incluyen:

- La responsabilidad ministerial dividida, producto de las ideas asistencialistas dirigidas más hacia el tratamiento y la segregación social de las personas con discapacidad que a la inclusión y la igualdad de oportunidades. Aquí, la provisión de los servicios educativos está a cargo de varios ministerios, que comparten la responsabilidad o, peor aún, operan de manera separada.

-La ausencia de legislación, políticas, metas y planes, reflejo del compromiso variable que los países tienen con la inclusión, son también resultado de la falta de articulación, ya que las leyes no siempre se concretan en políticas, o bien, se elaboran políticas, pero no se diseñan estrategias específicas. Además, cuando se establecen metas, estas son poco viables o se carecen de servicios de apoyo y protección social para concretarlas.

-Recursos insuficientes, que abarcan no solo los de tipo económico, sino también de infraestructura, como el bajo número de escuelas o instalaciones inadecuadas; los recursos humanos, ya sea porque hay pocos docentes o porque no están capacitados; y los materiales educativos.

Respecto a las barreras escolares, la OMS (2011) destaca las siguientes:

- La pedagogía y los planes de estudio, que comprenden currículos y métodos de enseñanza rígidos, material didáctico no accesible, estrategias de evaluación descontextualizadas y exámenes que privilegian el desempeño académico.

-La formación y los apoyos insuficientes para los maestros, que se relaciona con el problema de los recursos. En los lugares socialmente desfavorecidos, los docentes suelen tener un gran número de niños por clase; esto mengua los recursos para su formación en inclusión, reduce las posibilidades de contratar personal de apoyo y disminuye el tiempo para la atención de las necesidades de los alumnos.

-Las barreras físicas, que comprenden el acceso físico a los edificios escolares, mobiliario, equipos e instalaciones sanitarias inaccesibles y los trayectos entre las escuelas y los hogares de los estudiantes.

-Los rótulos, es decir, los estigmas que se atribuyen a las personas con discapacidad y que, en muchas ocasiones, resultan de los estudios de diagnóstico que se realizan para canalizar a los estudiantes a la educación especial o a otros servicios de apoyo, en los que, de nuevo, predomina el enfoque asistencialista.

-Las barreras actitudinales, en las que se superponen las ideas, el lenguaje y 
las prácticas elaboradas socialmente, que ven a la discapacidad como un castigo divino o creen que estos estudiantes no tienen capacidad para aprender, que los maestros no están obligados a atenderlos, o que deben ser remitidos a escuelas de educación especial. Estas barreras atañen por igual a profesores, estudiantes, directivos y padres de familia.

-Los actos de violencia, intimidación y abuso, en los que están presentes los estigmas y las creencias en torno a la discapacidad. Pueden ir desde la violencia verbal o física hasta la psicológica, como las amenazas o el aislamiento, y que son cometidos por alumnos, docentes u otros miembros del personal.

En la educación superior, varios autores (Hewett, Douglas, McLinden \& Keil, 2017; Moola, 2015; Moriña y Cotán, 2017; Wray, 2011) coinciden en que los estudiantes con discapacidad suelen enfrentar más dificultades que sus pares sin discapacidad y tienen que invertir una mayor cantidad de tiempo para superarlas. En este nivel, las barreras han sido un tema menos desarrollado comparado con los trabajos sobre la educación obligatoria; sin embargo, estas no difieren mucho de las ya señaladas.

En una investigación realizada en España sobre el acceso y la participación de las personas con discapacidad a la enseñanza superior de régimen general (Red2Red, 2013), se encontraron los siguientes tipos de barreras:

-Sociales, actitudinales y de comunicación, que incluyen: las bajas expectativas sobre la solvencia de las personas con discapacidad para cursar la educación superior; la carencia de información y sensibilización sobre el tema; la sobreprotección familiar; la depreciación profesional; la falta de instancias para la formación profesional; la poca comunicación entre los profesores y las oficinas de atención a los alumnos; y las ideas respecto a los ingresos económicos y la movilidad laboral, que restringen las posibilidades para que esta población estudie carreras largas.

-Arquitectónicas y espaciales, que comprenden: los problemas de desplazamiento entre las viviendas y las instituciones educativas; los costos del transporte adaptado; la poca accesibilidad de las instalaciones universitarias, el mobiliario y equipo; y la falta de un abordaje integral a las necesidades de los alumnos con discapacidad.

-Técnicas y humanas, que abarcan: la poca formación de los profesores sobre el tema de la discapacidad; la insuficiencia de personal especializado, como los intérpretes de lengua de señas, o la capacitación insuficiente para el personal de apoyo; y la ausencia de asistentes profesionalizados.

La incorporación a sus grupos y el establecimiento de vínculos con sus compañeros es otro problema, ya que, aunque las personas sean cordiales con ellos, al mismo tiempo pueden dudar de sus aptitudes y méritos para estar en la educación superior (OMS, 2011). Esto, además, puede ser una limitante para que ellos revelen su discapacidad, el denominado disclosure, y expresen sus necesidades ante sus compañeros, profesores o el personal universitario (Getzel, 2008). Las barreras actitudinales 
tienen una gran incidencia en el éxito académico de este sector de la población; no obstante, en la educación superior, las probabilidades de éxito disminuyen cuando se han tenido experiencias previas negativas.

Finalmente, en cuanto a las clases, trabajos como los de Fuller, Bradley y Healey (2004), Hopkins (2011), Moriña y Carballo (2018) y Moriña y Cotán (2017) han encontrado barreras, como la cantidad de temas considerados en los programas; las dificultades para adaptar los contenidos y métodos de enseñanza; la utilización de estrategias didácticas centradas en el profesor; la organización y los tiempos destinados para las actividades; las dificultades para participar en clases; el uso de criterios de evaluación y exámenes estandarizados; la falta de personal de apoyo, como lectores o tomadores de notas; y la escasez de materiales académicos accesibles.

\section{LA DÍADA BARRERAS-FACILITADORES}

Las barreras para la inclusión inciden en las oportunidades que las personas con discapacidad tienen para desarrollarse a plenitud en los distintos ámbitos de la vida social. Específicamente en el nivel superior, estas llegan a tener un peso importante en la trayectoria académica; la cantidad y el tipo de barreras son cuestiones que los estudiantes consideran al momento de escoger la carrera, los cursos y sus tutores (Hopkins, 2011).

No obstante, las barreras no surgen ni actúan de manera autónoma, sino que son el resultado acumulado de acciones colectivas. Como nos advierte Young (2001,) a propósito de las estructuras sociales, es un error pensar en ellas como "entidades independientes de los actores sociales, yaciendo pasivamente alrededor de ellos, facilitando o inhibiendo sus movimientos" [as entities independent of social actors, lying passively around them easing or inhibiting their movement] (traducción propia, p. 13).

Cuando hablamos de las barreras, en general, hacemos referencia a cosas muy concretas, como el transporte, la señalización, el acceso a los edificios, la formación de profesores, los recursos pedagógicos, las normas y las actitudes, entre otros. Sin embargo, ninguno de estos indicadores, por llamarlos de alguna manera, son barreras por sí mismos; se convierten en ellas a partir de la interacción entre las condiciones sociales y las capacidades individuales.

Entonces, si las barreras son construcciones sociales y no son independientes de los sujetos, las podemos cambiar, es decir, transformarlas en facilitadores. En la literatura especializada en inclusión no suele encontrarse una definición precisa de este término, porque la mayoría centra la discusión en el tema de las barreras. Al respecto, Wray (2011) comenta que, hasta ahora, se desconoce si lo anterior se debe a una mayor prevalencia de experiencias negativas sobre la discapacidad o simplemente a que esta es una línea de investigación poco explorada.

En la investigación de la que se deriva este artículo, entendemos los facilitadores como todos aquellos factores que favorecen la presencia, la participación y el aprendizaje de los estudiantes con discapacidad, y que resultan del reconocimiento de la diversidad humana. Comprenden la implementación de apoyos necesarios y ajustes pertinentes, e implican la redefinición de las leyes y normas, la redistribución de 
los recursos y la ampliación de las oportunidades educativas. Asimismo, nos interesa destacar tres aspectos que, desde nuestra perspectiva, caracterizan la relación barreras-facilitadores: la reversibilidad, el efecto de refuerzo y la extensibilidad.

La reversibilidad. Este es un rasgo que, de algún modo, hemos señalado antes. Como ocurre con otros factores que incrementan el riesgo de exclusión, las barreras y los facilitadores tienen un carácter sociohistórico; sus efectos en la vida de las personas no se producen de forma automática, sino por la combinación de diversas circunstancias tanto individuales como sociales (Gairín y Suárez, 2012).

En el ámbito educativo, dichas condiciones pueden remitirse a cuestiones internas a las instituciones, como la falta de recursos o de personal capacitado, o externas a ellas, como el nivel de ingresos o el capital cultural familiar. No obstante, justamente porque son una construcción colectiva, las barreras y los facilitadores son reversibles, es decir, sus fronteras son flexibles (Subirats, Gomà y Brugué, 2005). De este modo, la organización de los planes de estudio que es una barrera señalada con reiteración por los estudiantes con discapacidad, tanto por la cantidad de cursos que tienen que tomar por semestre como por los requerimientos para acreditarlos, se puede convertir en un facilitador para la permanencia y el logro educativo, si se hacen los ajustes necesarios.

No obstante, transformar las barreras en facilitadores demanda acciones concertadas entre las diferentes instancias educativas, así como entre los departamentos al interior de las instituciones (Terigi, 2014). De igual modo, supone comprender la complejidad de causas que están detrás de las barreras y la profundidad de sus alcances.

El efecto de refuerzo. Las barreras no solo son producto de procesos acumulados, sino que también generan consecuencias acumuladas. Aquí, otra vez, nos apoyamos en Young (2001), quien sostiene que, para comprender el efecto que ejercen los componentes estructurales en la acción social, hay que verlos en su conjunto, como los alambres de una jaula. Si los analizamos uno por uno, con dificultad podremos observar la forma en que favorecen las oportunidades de algunos individuos o grupos y restringen las de otros.

En ese sentido, las barreras se refuerzan mutuamente incrementando el riesgo de exclusión; por ejemplo, la falta de libros, materiales y equipos accesibles hace que los alumnos con discapacidad tengan muchas dificultades para cumplir las tareas y trabajos académicos, lo que, a la postre, afecta su nota al final del semestre. Además, esto puede significar gastos adicionales, como en el caso de los estudiantes con discapacidad visual o múltiple, que tienen que invertir en impresiones en Braille o en software especializado cuando las instituciones no cuentan con estos servicios.

Lo mismo ocurre con los facilitadores; por ejemplo, si las oficinas institucionales tuvieran protocolos claros para la realización de trámites escolares, no solo se ahorraría tiempo, sino que también se mejorarían las condiciones para que los alumnos con discapacidad pudieran seleccionar e inscribir los cursos que quieren tomar.

Finalmente, la identificación de las barreras no debe servir únicamente para denunciar lo que hace falta o lo que no están haciendo las universidades; como sugieren Echeita y Ainscow (2011), también deben entenderse como una guía para

Pérez. Entre barreras y facilitadores: las experiencias de los estudiantes universitarios con discapacidad Sinéctica 53 www.sinectica.iteso.mx 
identificar los puntos fuertes e implementar medidas que mejoren las prácticas, procesos y condiciones institucionales.

La extensibilidad. Este rasgo se refiere a los beneficios que podemos obtener al eliminar las barreras y ampliar los facilitadores. Uno de los argumentos más recurrentes cuando se solicitan ajustes o apoyos es que los costos que representan no se corresponden con las posibles ventajas, porque están dirigidos a una minoría.

Para Nussbaum (2007), detrás de este planteamiento hay una simplificación estadística, esto es, como la población con discapacidad es pequeña en comparación con la población sin discapacidad, la solución más práctica es que aquella se adapte a las condiciones existentes. Más importante aún, este tipo de razonamiento remite a un modelo de persona en el que pareciera que, a lo largo de toda su existencia, será autónoma, libre y estará en plenitud de sus capacidades (Nussbaum, 2007). Esta idea, señalada en numerosas ocasiones por los movimientos a favor de los derechos de las personas con discapacidad, tiene muy poco que ver con la realidad porque no existe un estándar de "normalidad" como tal (Shakespeare \& Watson, 2001) y porque en nuestras vidas hay momentos o etapas completas en las que somos dependientes de otros, como cuando tenemos un accidente y perdemos movilidad, o cuando somos bebés y requerimos del cuidado de nuestros padres.

De este modo, la eliminación de las barreras y la implementación de facilitadores pueden beneficiar a toda la comunidad; por ejemplo, retomando el tema de los protocolos para los trámites institucionales; aquí, las ventajas no solo serían para los estudiantes con y sin discapacidad, sino también para el personal administrativo, e incluso para los docentes.

\section{Metodología}

Lo que aquí presentamos es parte de una investigación financiada por el Programa de Apoyo a Proyectos de Investigación e Innovación Tecnológica, de la Universidad Nacional Autónoma de México (UNAM), cuyo objetivo es analizar los alcances que las políticas universitarias para la inclusión tienen en la presencia, participación y aprendizajes de los estudiantes con discapacidad, desde la perspectiva de los propios sujetos.

El método utilizado fue el estudio de caso, entendido como un proceso de indagación profundo, en nuestra investigación sobre los alumnos con discapacidad, así como el resultado de dicha indagación (Stake, 2003). Para delimitar el caso, establecimos las siguientes líneas temáticas: antecedentes institucionales sobre la inclusión; condiciones para la inclusión; información institucional sobre los estudiantes con discapacidad; diversidad de la oferta académica; y presencia y participación de las personas con discapacidad. Como se observa, las líneas fueron del tipo issue questions (Neiman y Quaranta, 2006), dado que nuestro principal interés era analizar las políticas educativas para la inclusión. Esto nos permitió seleccionar dos instituciones: la UNAM y la Universidad Juárez Autónoma de Tabasco (UJAT).

La UNAM ha sido pionera en la provisión de servicios para la población con discapacidad; por ejemplo, desde 1959, se dispone de una sala de tiflológico para personas ciegas o con baja visión y, en 2003, se emitieron los Lineamientos para dar 
atención a las personas con capacidades diferentes -que era la denominación que se usaba en esos momentos-. Un año después se fundó el Comité de Atención a las Personas con Discapacidad y, en 2013, la Unidad de Atención a Personas con Discapacidad. También, se han implementado medidas en materia de normativa y legislación, apoyos académicos y económicos, accesibilidad física y de información, actividades deportivas, culturales y de difusión (Pérez-Castro, 2016, 2019).

En la UJAT, el tema de la discapacidad se empezó a incorporar en los planes institucionales de desarrollo como parte de las políticas de atención a los grupos vulnerables, a partir del 2000, y como una estrategia para la inclusión de los sectores desfavorecidos, en 2004. El primer registro que se tiene de un estudiante con discapacidad es en 2008, en la carrera de Técnico Superior en Música (Palmeros, 2014). Hasta ahora, las principales medidas han sido las tutorías y mentorías para los estudiantes en desventaja académica y la accesibilidad física (Pérez-Castro, 2016).

Evidentemente, se trata de universidades con trayectorias distintas en materia de inclusión; no obstante, en ambas pudimos tener acceso a la información y, sobre todo, vincularnos con los estudiantes, factores que contribuyeron a su selección como parte de la investigación.

\section{Estrategias e instrumento de investigación}

En el estudio de caso cualitativo, los datos subjetivos son fundamentales, porque "lo que se llega a saber y comprender del caso se consigue mediante el análisis y la interpretación de cómo piensan, sienten y actúan las personas" (Simons, 2011, p. 21). Específicamente, aquí nos interesaba recuperar la valoración de los estudiantes con discapacidad respecto a las políticas universitarias para la inclusión, si se concretaban en estrategias y apoyos a lo largo de su trayectoria o si, por el contrario, abonaban muy poco a superar las barreras institucionales.

Para acercarnos a los sujetos, utilizamos la entrevista en profundidad. Elaboramos un guion conformado por siete dimensiones que, desde el enfoque social de la vulnerabilidad, se denominan dimensiones de la interacción humana (Wilches-Chaux, 1993): física, económica, técnica, institucional, social, cultural y natural. Estas se cruzaron con las categorías de presencia, participación y aprendizajes, que constituyen los tres grandes componentes de la inclusión educativa (Echeita y Ainscow, 2011).

El instrumento se piloteó con estudiantes de las universidades, se ajustaron las preguntas que no eran claras y se eliminaron aquellas que no aportaban información relevante. Para la selección de los sujetos, usamos un muestreo intencional a partir de dos criterios fundamentales: que se tratara de alumnos con discapacidad y que estuvieran en diferentes momentos de la trayectoria académica, esto es, ingresando a la carrera, a la mitad o a punto de terminarla y recién egresados.

Asimismo, dado que este método implica profundizar tanto en lo particular como en lo común del caso en cuestión (Stake, 2003), a la par de las entrevistas, recurrimos al análisis documental de las políticas, planes de desarrollo e información estadística sobre la población estudiantil con discapacidad, en las dos universidades. También, utilizamos otras fuentes, documentales y testimoniales, sobre 
las condiciones personales e institucionales. Con todo esto y con la información de las entrevistas, triangulamos los datos para asegurar la validez (Denzin, 2009) y, junto con la revisión teórica, construir las categorías para analizar los resultados.

\section{Los sujetos de estudio}

Realizamos un total de 20 entrevistas, 9 a estudiantes de la UJAT y 11 a estudiantes de la UNAM. La mitad de los entrevistados eran hombres y la otra mitad, mujeres, que se encontraban en diferentes momentos de su trayectoria educativa; 15 aún estaban estudiando, 1 había terminado los créditos y 4 acababan de egresar de la universidad.

Respecto a la edad, 8 tenían entre 20 y 24 años, 7 estaban entre los 25 y 29 años, y el resto tenían más de 30 años. Igualmente, buscamos abarcar diferentes tipos de discapacidad, de tal manera que 9 alumnos tenían discapacidad visual, 3 con baja visión y 6 eran personas ciegas, 7 estudiantes tenían discapacidad motriz, 3 discapacidad múltiple y 1 discapacidad auditiva. Por último, 3 de los entrevistados habían hecho sus estudios previos únicamente en escuelas especial, 7 cursaron una parte en el sistema especial y la otra en el sistema regular, y 10 habían estudiado solo en escuelas regulares.

\section{BARRERAS Y FACILITADORES EN UN GRUPO DE ESTUDIANTES UNIVERSITARIOS}

La información brindada por los estudiantes con discapacidad abarcó diferentes dimensiones de su vida en la universidad. Los entrevistados señalaron desde cuestiones estrictamente académicas hasta aspectos relacionados con su experiencia de la discapacidad. Para los fines de este artículo, nos enfocamos en las barreras y los facilitadores, y tomamos como base la distinción que hace French (2017) entre barreras estructurales, ambientales y actitudinales; las cruzamos con las otras clasificaciones que aquí hemos incluido para ampliar su capacidad explicativa.

Vale la pena aclarar que los nombres de los entrevistados fueron sustituidos por códigos, por ejemplo, E20-F-UNAM. En la primera parte, E corresponde a "estudiante" y 20, al número asignado para identificarlo; la segunda parte indica el género, en este caso femenino; y la tercera es la institución de adscripción.

\section{Las barreras estructurales}

En este nivel, la primera barrera con la que se enfrentan las personas con discapacidad es la normativa universitaria. Aunque la inclusión es un tema que se ha incorporado paulatinamente al debate y a las políticas de educación superior en México (Cruz y Casillas, 2017; Pérez-Castro, 2014, 2016), los reglamentos, sistemas y programas de apoyo institucionales están pensados para un tipo de estudiante "promedio", lo que impide ver que algunos pueden tener necesidades específicas.

Las barreras más recurrentes, de acuerdo con los entrevistados fueron:

-Trámites institucionales engorrosos (11). 
-Falta de información accesible sobre las carreras (8).

-Poca disponibilidad de becas y apoyos económicos (7).

En especial, se hizo hincapié en la diversidad de trámites contradictorios o poco claros, en los cuales la ayuda de los padres y compañeros de estudio ha sido fundamental:

Había veces que me decían, este trámite lo haces en secretaría escolar y en secretaría me decían, no pues es que lo tienes que hacer en la coordinación de tu carrera, y la coordinación de mi carrera estaba en el tercer piso [...] Entonces, le decía a mi papá: "Sube y diles que vas de mi parte" y le daba yo mi credencial. Creo que en un par de ocasiones tuve que firmar una carta de poderes, para que él hablara en mi nombre [E13-M-UNAM].

Sobre el tema de las becas, uno de los entrevistados comentó:

Recibo la beca de manutención, que es la federal, pero, cuando entré [a la universidad] tuve que pagar lo mismo que todos los demás. Afortunadamente, no era tan caro, pero para mi familia sí era un gasto grande [E2-M-UJAT].

Como se observa, se trata de barreras estructurales de tipo sistémico (OMS, 2011), resultado de la ausencia de una perspectiva incluyente en la legislación y políticas institucionales o de recursos insuficientes. En las dos universidades de nuestra investigación, la inclusión de los estudiantes con discapacidad es una temática presente en los planes de desarrollo (Graue, 2017; Piña, 2016); sin embargo, consideramos que hace falta traducirla a programas y estrategias específicos para las diferentes dependencias institucionales, las facultades o divisiones académicas y las carreras.

Las barreras estructurales impactan la inclusión en las aulas, así como el desempeño de los estudiantes con discapacidad. Las mencionadas por nuestros entrevistados no distan mucho de las que se abordan en otros estudios (Healey, Fuller, Bradley y Hall, 2006; OMS, 2011; Red2Red, 2013), como:

-Dificultades para ajustar los contenidos y las actividades de enseñanza (11).

-Formas y tiempos de evaluación poco flexibles (10).

-Programas educativos rígidos y con pocas posibilidades de reincorporación (9).

-Falta de preparación de los profesores sobre los temas de inclusión y discapacidad (7).

Respecto a las actividades en clases, una alumna comentó:

Han sido varias cosas. La primera es que hay cosas que no puedo hacer porque son demasiado visuales, las actividades en el salón. Tengo el material, pero pues es visual, son copias y aquí no hay material en Braille (E9-F-UJAT).

Las barreras en el aula tienden a multiplicarse, dependiendo del número de materias que se deben cursar, lo que puede llevar a la deserción o reprobación:

... son muchas materias y, entonces, todas te piden tareas para la semana que viene, te dan mucha información, o sea, sí se me complica un poco esa situación. Cuando en otras [carreras] he visto que máximo por semestre son seis. Yo llevo siete u ocho (E17F-UNAM). 
Evidentemente, en el rendimiento académico intervienen múltiples factores, como la formación escolar previa, el capital cultural y la capacidad para adaptarse a los valores y exigencias institucionales (Bonal y Tarabini, 2013); no obstante, se ha observado que los alumnos con discapacidad suelen enfrentar un mayor número de barreras, como los contenidos de los programas, el tipo de estrategias y materiales didácticos, el diseño de las tareas en aula y las formas de participación consideradas por los profesores (Fuller, Bradley y Healey, 2004; Hopkins, 2011; Moriña y Cotán, 2017), que, a la postre, pueden afectar su desempeño, aunque cuenten con suficientes conocimientos y habilidades para la educación superior (Riddell, Tinklin \& Wilson, 2005).

La evaluación fue otra barrera en la que coincidió la mitad de nuestros entrevistados. En la calificación final, los exámenes y trabajos por escrito suelen tener un peso importante, tanto en la UNAM como en la UJAT; no se tiene en cuenta que los alumnos con discapacidad visual o múltiple requieren asistentes de lectura y escritura cuando no disponen del lector de pantalla o no se les permite usarlo, ni tampoco se consideran tiempos pertinentes para que contesten los exámenes. Lo mismo ocurre con los ensayos finales. El problema es que, por una parte, las instituciones no disponen de este tipo de apoyos y, por otra, hay periodos establecidos para la entrega de las notas finales, los cuales, en general, dependen de los departamentos de servicios escolares $y$, aunque se pueden solicitar algunos ajustes, esto no siempre es posible.

Las medidas tomadas para enfrentar este problema son diversas; están aquellos que señalan que los maestros les asignaron una calificación desde el inicio del semestre para "ahorrarse" el esfuerzo o los que terminaron por reprobar la materia, hasta los que tuvieron el apoyo de sus profesores o coordinadores de carrera:

Sí, me tocó uno [maestro] que me decía: "Pues, yo ya tuve un compañero como tú y lo tuve que pasar", y me dijo: "Si tú vas a ser igual, pues desde ahorita te doy el ocho" (E10-F-UNAM).

Desde que entré a la universidad todo ha sido oral, nunca ha habido un examen escrito y yo le tengo que leer las respuestas al maestro. Al igual que, si ya se terminó el tiempo y me falta escribir una respuesta, ya ni modo, no tengo más tiempo (E3-M-UJAT).

El tema de la evaluación y los exámenes es una barrera que también reportan otras investigaciones, en las que no solo se ha analizado el impacto que esto tiene en el desempeño de los alumnos (Healey et al., 2006; Moriña y Cotán, 2017), sino también en el trabajo de los docentes, en especial cuando se deben hacer ajustes muy específicos (Moriña y Carballo, 2018).

Por supuesto, aquí intervienen diversos factores, como la normativa institucional, las estrategias de evaluación, la formación de los profesores, además de la disposición y comunicación entre estudiantes y maestros. Los resultados de nuestra investigación muestran experiencias favorables y desfavorables en las dos universidades.

... hay maestros que sí me esperan un poquito, porque me dicen: "No pues, necesitas un poquito más de tiempo, ya me traes el trabajo después". Pero algunos no, me dicen que de plano se los tengo que entregar rápido, al igual que los demás (E7-M-UJAT).

Sí, yo hablaba mucho con ellos [los maestros] y les explicaba mi situación de discapacidad y las dificultades que tenía con mi problema de la espalda. Y me daban más tiempo para hacer las cosas. Sí, eran muy conscientes en ese aspecto (E12-M-UNAM).

Pérez. Entre barreras y facilitadores: las experiencias de los estudiantes universitarios con discapacidad Sinéctica 53 www.sinectica.iteso. $m x$ 
Por último, en las barreras estructurales, el tipo de discapacidad es una variable que marca diferencias. En nuestros sujetos de estudio, aquellos con discapacidad múltiple y sensorial, ya sea visual o auditiva, fueron los que más señalamientos hicieron respecto a los problemas estructurales, tanto sistémicos como escolares.

\section{Las barreras ambientales}

Esta categoría comprende las barreras para la accesibilidad física, a la información y la carencia de personal especializado, como los intérpretes de lengua de señas o los asistentes de lectura. En nuestros entrevistados hubo algunas diferencias de acuerdo con el tipo de discapacidad. Los estudiantes con discapacidad motriz y algunos con discapacidad múltiple dijeron que la mayor parte de las barreras estaba en:

-El acceso a los salones, bibliotecas, centros de cómputo, laboratorios y oficinas (8).

-El mobiliario de las aulas (5).

... los edificios son algo deteriorados, entonces, cuando yo llegué [a la universidad], sí estaba el acceso a las rampas, pero estaban mal hechas, en el sentido de que algunas están muy inclinadas, se me dificultaba mucho al principio poder dominarlas y bajar sola (E4-F-UJAT).

... yo hubiera querido que cambiasen el salón al segundo piso, pero, la propia FES [Facultad de Estudios Superiores] no tiene instalaciones más que para la gente en silla de ruedas y eso es doloroso para los que usamos bastón o muletas. No está pensado para nosotros (E19-F-UNAM).

Por su parte, las personas con discapacidad visual hicieron hincapié en:

-La escasez de material bibliográfico accesible y sobre los contenidos de sus carreras (9).

- La carencia de equipos adaptados (6).

- La ausencia de personal de apoyo (5).

-La falta de señalética (5).

Casi todos [los materiales] eran como de lectura, bueno, a veces los PDF podían estar mal escaneados, entonces, el contraste sí podía ser un tema difícil para mi vista (E14-FUNAM).

No hay [equipos], yo tengo que traer mi lap [...] y también tengo un programa que es portable, que se instala en cualquier computadora. Eso es lo único que me ha facilitado, pero la universidad no. Incluso, tengo que enseñar al maestro cómo se usa, le explico cómo es y lo sensibilizo en esa parte de la tecnología ¿no? (E3-M-UJAT).

Llama la atención que, sin importar el tipo de discapacidad, varios de los entrevistados (13) coincidieron en la dificultad que les representa moverse en las instalaciones, por las distancias entre los edificios, los pasillos estrechos y las condiciones de las vías internas. Igualmente, la mayoría (15) comentó tener problemas para desplazarse de sus casas a la universidad por la falta de transporte accesible y en buen estado.

Pérez. Entre barreras y facilitadores: las experiencias de los estudiantes universitarios con discapacidad Sinéctica 53 www.sinectica.iteso.mx 
Cabe señalar que, en este tipo de barreras, hubo variaciones por institución. En la UNAM, de acuerdo con el testimonio de los estudiantes, hay una mayor disponibilidad de apoyos, como el transporte interno, las impresiones en Braille y la posibilidad de solicitar un cambio de aula. En la UJAT, la ayuda ha consistido, principalmente, en la asignación de salones en planta baja.

Las barreras ambientales, en especial las físicas, han sido las que más se han abordado en la educación superior. En algunos países, como Estados Unidos, Dinamarca, Reino Unido, Francia, Irlanda, Noruega y República Checa, se han elaborado leyes que obligan a las instituciones a hacer adaptaciones para garantizar el acceso a las personas con discapacidad (OCDE, 2011). En América Latina no siempre se cuenta con legislación tan específica, pero hay leyes a favor de la inclusión de las personas con discapacidad o en contra de la discriminación en Argentina, Brasil, Chile, Costa Rica, Ecuador, Guatemala, México, Perú, República Dominicana y Venezuela (Pérez-Castro, 2019).

Sin embargo, aún existen muchas barreras ambientales en las instituciones de educación superior, aun en los países desarrollados. En algunos casos, como advierte Wray (2011), estas intervienen desde antes del ingreso, ya que es un aspecto que los alumnos sopesan al seleccionar la universidad, mientras que, en otros, las consecuencias se reflejan en la permanencia y el desempeño académico.

\section{Las barreras actitudinales}

Estas son tal vez las barreras que mayor alcance tienen en la inclusión de las personas con discapacidad en la educación superior. Para Tinklin y Hall (1999), la calidad de las experiencias de estos estudiantes depende, en gran medida, de la conciencia de todo el personal y nosotros agregaríamos que también del conocimiento sobre el tema.

Nuestros sujetos de estudio refirieron barreras como

-Las actitudes negativas de los profesores (9).

-La poca sensibilización del personal administrativo y de apoyo (7).

-Las actitudes negativas de los compañeros (6).

Sobre las actitudes de los maestros, en ambas universidades, las opiniones se dividieron entre aquellos que señalaron que estas eran poco favorables, debido al desconocimiento que tenían sobre la discapacidad, a su limitada formación para realizar los ajustes necesarios o simplemente al descuido, y los que apuntaron que, a pesar de que no tener información, la mayoría se mostraba dispuesta a ayudarlos.

Es que algunos maestros, cuando hablan, se olvidan de lo que les acabo de decir, no oigo bien [...] yo me pongo enfrente y dependiendo de su forma de hablar, puedo entenderlo y cuando no, les pregunto a algunos compañeros (E5-M-UJAT).

Una persona que tiene que ser hospitalizada regularmente comentó:

Yo tengo que hablar con ellos [los profesores] y unos sí te dicen: "Vamos a ver el problema, vas a tener esta situación, pero vamos a adecuarnos así y así". Y otros sí te dicen: "No pues si no vienes te amolaste". Depende mucho del profesor (E18-M-UNAM). 
Como ocurre con las barreras estructurales, las normas y reglas del régimen académico acotan la labor de profesores y estudiantes (Baquero, Terigi, Toscano, Brisciol y Sburlatti, 2009), pero, muchas veces, los márgenes de acción pueden flexibilizarse a través de la comunicación que establezcan alumnos, maestros y autoridades institucionales. Conocer y aceptar la discapacidad, plantear con claridad los apoyos o ajustes requeridos, aprender a definir y ajustar las metas académicas, y distinguir lo que se puede hacer de manera autónoma y cuándo se requiere la ayuda de otros son algunas de las habilidades que favorecen el éxito académico de estas personas (Getzel, 2008; Getzel y Thoma, 2008).

No obstante, lo anterior es insuficiente sin la contraparte institucional, es decir, si la inclusión no forma parte de la política y los programas universitarios:

Construir una amplia inclusión institucional implica la asociación y la colaboración con todas las partes interesadas, a lo largo de todos los grupos de usuarios de las IES, incluidas las facultades académicas, los departamentos educativos, los servicios de apoyo a la discapacidad, los grupos de representación estudiantil y otras funciones. [Building institutional wide inclusion involves partnership and collaboration with all stakeholders across all HEI user groups including academic faculties, education departments, disability support services, student representative groups, and other functions.] (Traducción propia, AHEAD, 2017, p. 17.)

La participación de todos los departamentos es fundamental para la eliminación de las barreras y la implementación de los facilitadores. En nuestra investigación, los alumnos de ambas universidades destacaron la poca sensibilización que, en ocasiones, tiene el personal administrativo y de apoyo respecto a sus necesidades y que se manifiesta en la falta de información o de información contradictoria sobre los trámites a realizar, la mala atención o la indiferencia.

En cuanto a los facilitadores, la mitad de los entrevistados, tanto de la UNAM como de la UJAT, destacó el papel de los coordinadores de carrera y los profesores, de quienes han recibido ayuda para hacer los trámites, planear la trayectoria escolar o tramitar algún apoyo:

No sé si tienen [los maestros] una formación académica que hablen sobre discapacidad, pero, al menos, los que he conocido en mi carrera, tienen una formación humana, para poder enfrentar ese tipo de situaciones, porque mi carrera habla sobre diversidad social. Entonces, si se entiende a la discapacidad como un aspecto que forma parte de la diversidad, ellos tienen todas las herramientas para poder incluirlo (E14-F-UNAM).

Mi tutora y los maestros siempre han apoyado y siempre están al pendiente: “¿Ya hiciste eso?, ¿ya buscaste estos papeles?, ¿ya fuiste a la oficina? Si no puedes, avisa para que nosotros te apoyemos en tiempo y forma" (E1-M-UJAT).

En la construcción de instituciones educativas inclusivas, la formación y la toma de conciencia es buen punto de partida; sin embargo, a la par, se requiere desarrollar una cultura organizacional que valore positivamente la diversidad y fomentar prácticas que dinamicen los recursos humanos (Ainscow, 2012).

Por otra parte, para la mayoría de los entrevistados (17), la colaboración de sus amigos y compañeros de estudio fue primordial para su vida académica y social en la universidad. De hecho, después de su familia, ellos han sido el grupo de apoyo más importante. Su ayuda va desde notas en clases y búsqueda de materiales hasta la movilidad e incorporación a las actividades recreativas. Sin embargo, lo anterior no ha estado exento

Pérez. Entre barreras y facilitadores: las experiencias de los estudiantes universitarios con discapacidad Sinéctica 53 www.sinectica.iteso.mx 
de problemas. Algunos estudiantes reconocieron que, a la vez, han tenido que enfrentar la resistencia de ciertos compañeros que piensan que quizá no tienen las suficientes habilidades académicas y que trabajar con ellos les quitará tiempo, que terminarán absorbiendo sus deberes o, incluso, que afectará en sus calificaciones:

Hay integrantes que no quieren trabajar conmigo y hay integrantes que quieren trabajar conmigo. Es que ellos solo quieren estar con los mejores [...] Entonces, me quedo esperando, hasta que ellos se arrepienten solitos, si me necesitan o no me necesitan, y me doy cuenta que me quieren integrar (E5-M-UJAT).

Pues, yo creo que sí, todavía me siento un poquito, a veces, pues sí un poquito desplazada. Pero no es mucho, porque como que ya los compañeros y compañeras tienen ya otra visión de las personas con capacidades diferentes, ya no tanto la mirada discriminativa (E16-F-UNAM).

Si bien el desarrollo de redes de colaboración y de amigos en las instituciones educativas es un proceso de aprendizaje para todos los alumnos, con o sin discapacidad, para los primeros esto puede representar retos adicionales. Se necesitan más elementos sobre la relación entre los estudiantes con y sin discapacidad a fin de diseñar estrategias que favorezcan el trabajo colegiado y la relación entre ellos.

Al respecto, Liasidou (2014) plantea que las barreras actitudinales de los estudiantes sin discapacidad no han sido un tema recurrente en la investigación, a pesar de que es un factor importante para la inclusión o exclusión de estas personas.

Este parámetro se omite ocasionalmente cuando se discuten los problemas para mejorar la participación en la educación superior y genera inquietudes importantes sobre la necesidad de poner un énfasis más pronunciado en la comprensión de las experiencias educativas generales de este grupo de estudiantes. [This parameter is occasionally omitted when discussing issues of enhancing one's participation in higher education and raises significant concerns about the necessity of placing a more pronounced emphasis on understanding the overall educational experiences of this group of students.] (Traducción propia, p. 126.)

\section{CONSIDERACIONES FINALES}

Las barreras y los facilitadores constituyen una de las diferentes vías para acercarse a la inclusión (y exclusión) de las personas con discapacidad en la educación superior. Sus efectos son resultado de la convergencia entre las acciones colectivas, las acciones individuales y las condiciones sociales, y se manifiestan en diferentes dimensiones de la vida académica y social de los estudiantes.

En nuestra investigación, las barreras estructurales, tanto las de tipo pedagógico como sistémicas, fueron las más señaladas por los alumnos; les siguieron las relacionadas con la accesibilidad de la información, las actitudinales y las de accesibilidad física. No es extraño que las barreras pedagógicas hayan obtenido el mayor número de respuestas ni tampoco que, como parte de ellas, se hayan mencionado cuestiones referentes a los contenidos y las actividades de enseñanza, la evaluación, la estructura de los programas educativos y la formación de los profesores. De hecho, coincide con otras investigaciones (Moola, 2015; Healey et al., 2006; Wray, 2011), que muestran que estos aspectos intervienen de modo significativo en la trayectoria académica de los estudiantes, e incluso inciden en su decisión de ingresar o no la universidad, la selección de la institución y de la carrera. 
A esto se suman las barreras sistémicas, que ocuparon el segundo lugar de respuesta, y que se refieren a la carencia de normativa, políticas, programas y recursos institucionales para favorecer los procesos de inclusión. Entre los aspectos señalados estuvieron: los trámites institucionales, la falta de información accesible sobre la oferta académica y la disponibilidad de becas y apoyos económicos.

Si cruzamos estas barreras con las pedagógicas, podríamos decir que las oportunidades educativas de estas personas son bastante acotadas, ya que, como vemos, todas ellas remiten a la dimensión estructural-institucional y, además, son barreras cuyos efectos de refuerzo tiene enormes implicaciones en el ingreso, la permanencia y el egreso de la educación superior.

No obstante, las barreras, al igual que otros factores de riesgo social, pueden ser reversibles y es aquí en donde entran en juego los facilitadores. Tanto en el ámbito pedagógico como en el actitudinal, los estudiantes señalaron algunas acciones que, en su momento, les ayudaron a superar los obstáculos. En todas ellas, el factor humano fue fundamental, como el diálogo, la disponibilidad de los profesores y coordinadores de carrera, y los vínculos con los compañeros.

Esto es valioso porque habla del compromiso de los diferentes sujetos educativos, estudiantes con o sin discapacidad, maestros y personal administrativo. Sin embargo, tiene que haber un correlato con la parte institucional, esto es, se requieren políticas, programas, estrategias y fuentes de financiamiento que contribuyan a consolidar la perspectiva de la inclusión en la institución (AHEAD, 2017), así como del trabajo coordinado de las diferentes instancias y departamentos universitarios (Terigi, 2014). En suma, como proponen Booth y Ainscow (2002), se necesita transformar las culturas, las políticas y las prácticas.

Finalmente, queremos insistir en el efecto de refuerzo y el carácter extensivo de los facilitadores y las barreras. Varios de los asuntos aquí señalados son problemas que enfrentan todos los alumnos con o sin discapacidad. Implementar medidas que poco a poco transformen las barreras en facilitadores mejorará los aprendizajes y las experiencias de toda la comunidad estudiantil y permitirá construir las bases para desarrollar instituciones educativas incluyentes.

\section{REFERENCIAS BIBLIOGRÁFICAS}

Ainscow, M. (2012). Haciendo que las escuelas sean más inclusivas: lecciones a partir del análisis de la investigación internacional. Revista de Educación Inclusiva, vol. 5, núm. 1, pp. 39-49. Recuperado de http://www.revistaeducacioninclusiva.es/index.php/REI/article/view/220/214

Association for Higher Education Access \& Disability (AHEAD) (2017). Inclusive education. Position paper: A road map for disability support in higher education in Ireland. Dublín. Recuperado de https://www.ahead.ie/positionpaper

Baquero, R., Terigi, F., Toscano, A., Brisciol, B. y Sburlatti, S. (2009). Variaciones del régimen académico en escuelas medias con población vulnerable. Un estudio de casos en el área metropolitana de Buenos Aires. Revista Iberoamericana sobre Calidad, Eficacia y Cambio en Educación, vol. 7, núm. 4, pp. 293-319. Recuperado de https://revistas.uam.es/index.php/reice/article/ view/5394/5833 
Bennett, L., Ray, K. \& Wilson, T. (2016). Addressing barriers to work for disabled people and those with long term health conditions in Brighton \& Hove. Leicester: National Learning and Work Institute. Recuperado de https://ec.europa.eu/epale/sites/ epale/files/addressing_barriers_to_employment_for_disabled_people.pdf

Bonal, X. y Tarabini, A. (2013). De la educación a la educabilidad: una aproximación sociológica a la experiencia educativa del alumnado en situación de pobreza. Praxis Sociológica, núm. 17, pp. 67-88. Recuperado de http://ww.gepsuab.cat/sites/default/files/publicacions-adjunts/praxis17-05.pdf

Booth, T. \& Ainscow, M. (2002). Index for inclusion. Developing learning and participation in schools. Bristol: CSIE.

Cruz, R. y Casillas, M. (2017). Las instituciones de educación superior y los estudiantes con discapacidad en México. Revista de la Educación Superior, vol. 46, núm. 181, pp. 37-53. Recuperado de http://publicaciones.anuies.mx/pdfs/ revista/Revista181_S2A3ES.pdf

Denzin, N. (2009). The research act. A theoretical introduction to sociological methods. Nueva Jersey: Transaction Publishers.

Echeita, G. y Ainscow, M. (2011). La educación inclusiva como derecho. Marco de referencia y pautas de acción para el desarrollo de una revolución pendiente. Tejuelo, vol. 12, núm. 1, pp. 26-46. Recuperado de https://mascvuex. unex.es/revistas/index.php/tejuelo/ article/view/2497

French, S. (2017). Disabled people and employment. A study of the working lives of the visually impaired physiotherapists. Londres: Routledge.

Fuller, M., Bradley, A. \& Healey, M. (2004). Incorporating disabled students within an inclusive higher education environment. Disability \& Society, vol. 19, núm. 5, pp. 455-468. Recuperado de https://www.tandfonline.com/doi/ full/10.1080/0968759042000235307

Gairín, J. y Suárez, C. (2012). La vulnerabilidad en la educación superior. En J. Gairín, D. Rodríguez-Gómez y D. Castro (coords.). Éxito académico de colectivos vulnerables en entornos de riesgo en Latinoamérica (pp. 39-62). Madrid: Wolters Kluwer.

Getzel, E. (2008). Addressing the persistence and retention of students with disabilities in higher education: Incorporating key strategies and supports on campus. Exceptionality: A Special Education Journal, vol. 16, núm. 4, pp. 207219. doi: 10.1080/09362830802412216

Getzel, E. \& Thoma, C. (2008). Experiences of college students with disabilities and the importance of self-determination in higher education settings. $\mathrm{Ca}$ reer Development for Exceptional Individuals, vol. 3, núm. 2, pp. 77-84. doi: $10.1177 / 0885728808317658$

Graue, E. (2017). Plan de Desarrollo Institucional 2015-2019. México: UNAM. Recuperado de http://www.rector.unam.mx/doctos/PDI-2015-2019.pdf

Grover, C. \& Piggott, L. (2015). Disabled people, work and welfare. En C. Grover \& L. Piggott. Disabled people, work and welfare. Is employment really the answer? (pp. 1-22). Bristol: Policy Press/University of Bristol.

Healey, M., Bradley, A., Fuller, M. \& Hall, T. (2006). Listening to students: The experiences of disabled students of learning at university. En M. Adams \& S. Brown (eds.). Towards inclusive learning in higher education: Developing curricula for disabled students (pp. 32-43). Londres: Routledge.

Hewett, R., Douglas, G., McLinden, M. \& Keil, S. (2017). Developing an inclusive learning environment for students with visual impairment in higher education: Progressive mutual accommodation and learner experiences in the 
United Kingdom. European Journal of Special Needs Education, vol. 32, núm. 1, pp. 89-109. doi: 10.1080/08856257.2016.1254971

Hopkins, L. (2011). The path of least resistance: a voice-relational analysis of disabled students experiences of discrimination in English universities. International journal of inclusive education, 15 (7), 711-727. doi: 10.1080/13603110903317684

Hughes, B. y Paterson, K. (2008). El modelo social de discapacidad y la desaparición del cuerpo. Hacia una sociología del impedimento. En L. Barton (comp.). Superar las barreras de la discapacidad (pp.107-123). Madrid: Morata.

Hunt, P. (2017). A critical condition. En Boy Joys (ed.). Disability, space, architecture. A reader (pp. 33-41). Londres: Routledge.

Liasidou, A. (2014). Critical disability studies and socially just change in higher education. British Journal of Special Education, vol. 41, núm. 2, pp. 120-135. doi: 10.1111/1467-8578.12063

Moola, F. (2015). The road to the Ivory Tower: The learning experiences of students with disabilities at the University of Manitoba. Qualitative Research in Education, vol. 4, núm. 1, pp. 45-70. doi: 10.4471/qre2015.56

Moriña, A. y Carballo, R. (2018). Profesorado universitario y educación inclusiva: respondiendo a sus necesidades de formación. Psicología Escolar e Educacional, número especial, pp. 87-95. doi: 10.1590/2175-3539/2018/053

Moriña, A. y Cotán, A. (2017). Educación inclusiva y enseñanza superior desde la mirada de estudiantes con diversidad funcional. Revista Digital de Investigación en Docencia Universitaria, vol. 11, núm. 1, pp. 19-35. Recuperado de https://revistas.upc.edu.pe/index.php/docencia/article/view/528

Neiman, G. y Quaranta, G. (2006). Los estudios de caso en la investigación sociológica. En I. Vasilachis (coord.). Estrategias de investigación cualitativa (pp. 213-237). Barcelona: Gedisa.

Nussbaum, M. (2007). Las fronteras de la justicia. Barcelona: Paidós.

OCDE (2011). Inclusion of students with disabilities in tertiary education and employment. París.

Oliver, M. (1996). Defining impairment and disability: Issues at stake. En C. Barnes \& G. Mercer (eds.). Exploring the divide: Ilness and disability (pp. 39-54). Leeds: Disability Press.

OMS (2011). Informe mundial sobre la discapacidad. Ginebra: OMS/BM.

OMS (2001). Clasificación internacional del funcionamiento, de la discapacidady la salud. Madrid: OMS/OPS/MTAS/IMSERSO.

ONU (2006). Convención sobre los Derechos de las Personas con Discapacidad. Nueva York. Recuperado de http://www.un.org/disabilities/documents/ convention/convoptprot-s.pdf

Palmeros, G. (2014). Prácticas y experiencias en la División Académica de Educación y Artes de la Universidad Juárez Autónoma de Tabasco. En J. Gairín, G. Palmeros y A. Barrales. Universidad y colectivos vulnerables (pp. 131-139). México: Ediciones del Lirio.

Pérez-Castro, J. (2019). La inclusión de los estudiantes con discapacidad en dos universidades públicas mexicanas. Innovación Educativa, vol. 19, núm. 79, pp. 145-170. Recuperado de https://www.ipn.mx/assets/files/innovacion/ docs/Innovacion-Educativa-79/La-inclusion-de-los-estudiantes-con-discapacidad-en-dos-universidades.pdf 
Pérez-Castro, J. (2016). La inclusión de las personas con discapacidad en la educación superior en México. Sinéctica, Revista Electrónica de Educación, núm. 46. Recuperado de https://sinectica.iteso.mx/index.php/SINECTICA/article/view/614/641

Pérez-Castro, J. (2014). Can Mexico achieve an inclusive education? An analysis of higher education policies of recent decades. American International Journal of Social Science, vol. 3, núm. 6, pp. 77-84. Recuperado de http://www. aijssnet.com/journals/Vol_3_No_6_November_2014/10.pdf

Piña, J. (2016). Plan de Desarrollo Institucional 2016-2020. Villahermosa: UJAT. Recuperado de http://www.archivos.ujat.mx/2016/rectoria/PDI_FINAL2020.pdf

Red2Red (2013). Discapacidad, estudios superiores y mercado de trabajo. Barreras de acceso y repercusión en la inserción laboral. Madrid: Red2Red Consultores.

Riddell, S., Tinklin, T. \& Wilson, A. (2005). Disabled students in higher education. Perspectives on widening access and changing policy. Nueva York: Routledge.

Serrano, C., Ramírez, C., Abril, J., Ramón, L., Guerra, L. y Clavijo, N. (2013). Barreras contextuales para la participación de las personas con discapacidad física. Revista de la Universidad Industrial de Santander. Salud, vol. 45, núm. 1, pp. 41-51. Recuperado de https://revistas.uis.edu.co/index.php/revistasaluduis/article/view/3299/3499

Shakespeare, T. (2013). The social model of disability. En Lennard Davis (ed.). The disability studies reader (4a ed.) (pp. 214-221). Nueva York: Routledge.

Shakespeare, T. \& Watson, N. (2001). The social model of disability. An outdated ideology? En S. N. Barnatt y B. M. Altman (eds.). Exploring theories and expanding methodologies: Where we are and where we need to go. Research in Social Science and Disability, vol. 2 (pp. 9-28). Amsterdam: Emerald Group Publishing Limited.

Shields, N. \& Synnot, A. (2016). Perceived barriers and facilitators to participation in physical activity for children with disability: a qualitative study. $B M C$ Pediatrics, vol. 16, núm. 9. Recuperado de https://www.ncbi.nlm.nih.gov/ pmc/articles/PMC4717582/pdf/12887_2016_Article_544.pdf

Simons, H. (2011). El estudio de caso: teoría y práctica. Madrid: Morata.

Simpson, J. (2009). Inclusive information and communication technologies for people with disabilities. Disability Studies Quarterly, vol. 29, núm. 1. Recuperado de http://dsq-sds.org/article/view/167/167

Stake, R. (2003). Case studies. En N. K. Denzin \& Y. S. Lincoln (eds.). Strategies of qualitative inquiry (2a. ed.) (pp. 134-164). Thousand Oaks: Sage.

Subirats, J., Gomà, R. y Brugué, J. (2005). Análisis de los factores de exclusión social. Cataluña: Generalitat de Catalunya-Fundación BBVA.

Swain, J. \& French, S. (2014). International perspectives on disability. En J. Swain, S. French, C. Barnes \& C. Thomas (eds.). Disabling barriers -enabling environments (3a. ed.) (pp. 45-52). Londres: Sage.

Terigi, F. (2014). La inclusión como problema de las políticas educativas. En María del C. Feijoó y M. Poggi (coords.). Educación y políticas sociales. Sinergias para la inclusión (pp. 217-234). Buenos Aires: IIPE-Unesco.

Thomas, C. (2014). Disability and impairment. En J. Swain, S. French, C. Barnes \& C. Thomas (eds.). Disabling barriers -enabling environments (3a. ed.) (pp. 9-16). Londres: Sage. 
Tinklin, T. \& Hall, J. (1999). Getting round obstacles: disabled students' experiences in higher education in Scotland. Studies in Higher Education, vol. 24, núm. 2, pp. 183-194. doi: 10.1080/03075079912331379878

Wilches-Chaux, G. (1993). La vulnerabilidad global. En A. Maskrey (comp.). Los desastres no son naturales. San José de Costa Rica: Red de Estudios Sociales en Prevención de Desastres en América Latina.

Wray, M. (2011). Disabled learners and barriers to higher education. Warwick: Coventry and Warwickshire Aimhigher.

Young, M. (2001). Equality for whom? Social groups and judgments of injustice. The Journal of Political Philosophy, vol. 9, núm. 1, pp. 1-18. Recuperado de https://onlinelibrary.wiley.com/doi/epdf/10.1111/1467-9760.00115 